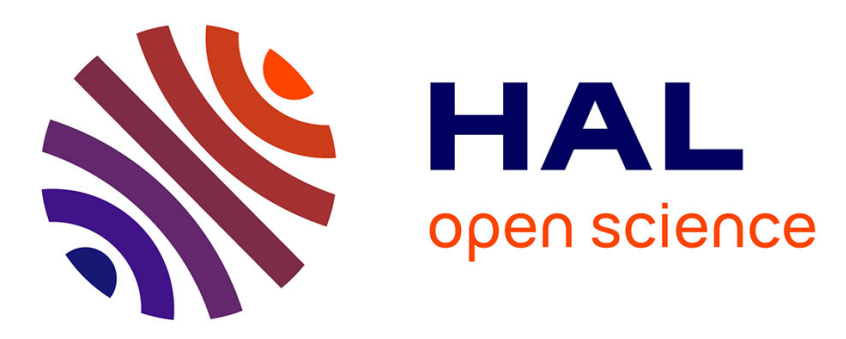

\title{
4D compressive sensing holographic microscopy imaging of small moving objects
}

Alexey Brodoline, Nitin Rawat, Daniel Alexandre, Nicolas Cubedo, Michel

Gross

\section{- To cite this version:}

Alexey Brodoline, Nitin Rawat, Daniel Alexandre, Nicolas Cubedo, Michel Gross. 4D compressive sensing holographic microscopy imaging of small moving objects. Optics Letters, 2019, 44 (11), pp.2827. 10.1364/OL.44.002827 . hal-02189448

\section{HAL Id: hal-02189448 \\ https://hal.science/hal-02189448}

Submitted on 19 Jul 2019

HAL is a multi-disciplinary open access archive for the deposit and dissemination of scientific research documents, whether they are published or not. The documents may come from teaching and research institutions in France or abroad, or from public or private research centers.
L'archive ouverte pluridisciplinaire HAL, est destinée au dépôt et à la diffusion de documents scientifiques de niveau recherche, publiés ou non, émanant des établissements d'enseignement et de recherche français ou étrangers, des laboratoires publics ou privés. 


\title{
4D compressive sensing holographic microscopy imaging of small moving objects
}

\author{
Alexey Brodoline ${ }^{1}$, Nitin Rawat $^{1,3}$, Daniel Alexandre ${ }^{1}$, Nicolas Cubedo $^{2}$, and Michel Gross ${ }^{1, *}$ \\ ${ }^{1}$ Laboratoire Charles Coulomb (L2C), Univ Montpellier, CNRS, Montpellier, France. \\ ${ }^{2}$ Mécanismes Moléculaires dans les Démences Neurodégénératives (MMDN), Univ Montpellier, INSERM, Montpellier, \\ France. \\ ${ }^{3}$ Electrical $\& 3$ Computer Engineering Department, University of Connecticut, USA \\ *Corresponding author:michel.gross@umontpellier.fr
}

\begin{abstract}
We show that compressive sensing (CS) calculations are very efficient to reconstruct in 3D sparse objects whose 2D hologram has been recorded by digital holographic microscopy. The method is well adapted to image small scattering objects moving within a larger motionless object. This situation corresponds to red blood cells (RBCs) circulating in the vascular system of a zebrafish (Danio rerio) larva. RBCs positions are imaged in 3D from a single hologram, while the RBCs trajectories, i.e. the perfused blood vessels, are imaged from a sequence of holograms. With respect to previous work (Donnarumma et al., Opt. express, 24, 26887, 2016), we get a gain of $\sim 500$ in calculation speed.
\end{abstract}

Citation: Alexey Brodoline, Nitin Rawat, Daniel Alexandre, Nicolas Cubedo, and Michel Gross, "4D compressive sensing holographic microscopy imaging of small moving objects," Opt. Lett. 44, 2827-2830 (2019) 
Digital holography (DH) captures the complex field scattered by an object, rather than its intensity, giving in this way 3D information on amplitude and phase. The recording of a hologram involves a semiconductorbased 2D imager (camera), while the reconstruction is performed numerically by a computer [19]. Most of the time, the field retrieval is done by retropropagating the hologram recorded on the $2 \mathrm{D}$ sensor within a series of planes adjacent to the object. In this way a $3 \mathrm{D}$ image is obtained.

It should be noted that this image corresponds to the field scattered by the object and is thus different from the object itself (i.e. from the 3D scattering density of the object). Indeed, as shown by Atlan et al. [1], the reconstructed image of a point object (ex: a gold bead of 100 $\mathrm{nm}$ diameter) obtained by digital holographic microscopy (DHM) is a cone whose apex corresponds to the position of the bead and whose opening angle corresponds to the numerical aperture of the microscope objective.

It is however possible to reconstruct a $3 \mathrm{D}$ image of the object by recording a series of holograms for different directions of illumination, and by performing a tomographic reconstruction that calculates in $3 \mathrm{D}$ the optical index distribution of the object $[8,21]$. However, because it requires to record a large number of holograms ( $\geq 100$ ), tomography only applies to immobile objects.

In the case where there is a basis in which the 3D scattering density is sparse enough, it is also possible to reconstruct a 3D image of the object from a single hologram by compressive sensing (CS) [11,3]. This point has been demonstrated by Brady et al. [2] who have imaged by holography an immobile object whose representation is sparse in the wavelet basis. Since this work, DH coupled to the CS has given rise to many developments. We can mention in particular compressive in-line holography [9], compressive Fresnel holography [17], offaxis frequency shifting holography [15], millimeter wave compressive holography [7], off-axis holography of diffuse objects [4], recovery of an image in low illumination conditions [14] or video-rate microscopic tomography [12].

In this letter, we show that CS digital holography is well adapted to image small scattering objects moving within a larger motionless object. Indeed, in the basis of the direct 3D space (i.e. $x, y, z$ ), the $3 \mathrm{D}$ scattering density of the moving objects is often sparse. This situation corresponds for example to the case of red blood cells (RBCs) circulating in a zebrafish (Danio rerio) larva, imaged by digital holographic microscopy (DHM) [10]. By performing CS reconstruction from a single hologram, we obtained an instantaneous 3D image of the objects (the RBCs). By performing a series of reconstructions from a hologram sequence, we obtained a $3 \mathrm{D}$ image of the trajectories of the objects (i.e. the blood vessels).

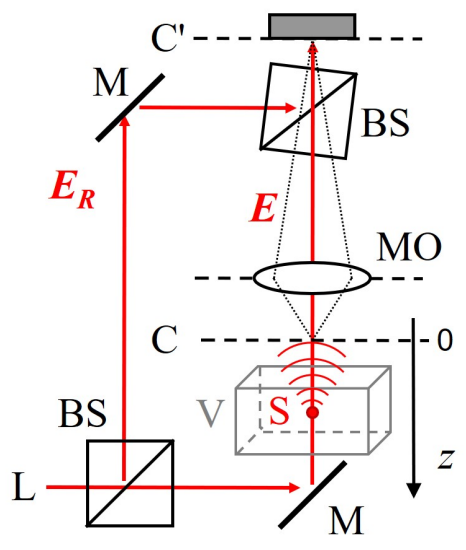

Figure 1: (a) Typical holographic microscopy setup. L: laser, C': camera, BS: beam splitter, M: mirror, MO: microscope objective, $\mathrm{C}$ : conjugate plane of plane $\mathrm{C}^{\prime}$ by objective $\mathrm{MO}, \mathrm{V}$ : imaged volume, $\mathrm{S}$ : a diffuser, $E$ : object field, $E_{R}$ : reference field.

To illustrate the relationship between 3D holographic reconstruction and CS, let us consider an experiment in which small moving objects are imaged with a typical transmission DHM setup (see Fig. 1). The objects moving in a volume $\mathrm{V}$, are illuminated by a laser $\mathrm{L}$ and scatter light in all the directions. The light is collected by the microscope objective $\mathrm{MO}$ and imaged on the camera, in plane $C^{\prime}$. Here, the field $E$ scattered by the object, interferes with the reference field $E_{R}$. The camera records the resulting interferogram $I=\left|E+E_{R}\right|^{2}$. Moving objects were selected by considering sequences of interferograms $I_{m}$ with $m=0,1,2 \ldots$ and by calculating the hologram $H_{C^{\prime}}$ in plane $\mathrm{C}^{\prime}$ by combining the $I_{m}$ with coefficients whose sum is zero (for example $\left.H_{C^{\prime}}=\left(I_{m}+I_{m-1}\right)-\left(I_{m-2}+I_{m-3}\right)\right)$. By this way, we subtracted the immobile background. This approach is very similar to the one of Schwerte et al. [20] who highlighted blood circulation in zebrafish larvae by calculating differences of successive frames in white light video movies.

The experiment is carried out in off-axis geometry. By filtering in the Fourier space [6], we can select the +1 order component of the hologram, corresponding to the $E E_{R}^{*}$ term of $I=\left|E+E_{R}\right|^{2}$ (where $E$ is the field scattered by the moving objects in the camera plane $C^{\prime}$ ). By considering that the reference field is flat field $\left(\left|E_{R}\right| \simeq 1\right.$ ), we thus calculate the field $E$. By eliminating the phase 
effects related to the aberrations of the MO objective [5], to the off-axis configuration and to the curvature of the reference wave [22], we get the field $E_{C}(x, y)$ in the plane $C$, conjugate of the plane $C^{\prime}$ by the objective MO. We get as well the field $\tilde{E}_{C}$ in the Fourier space: $\tilde{E}_{C}\left(k_{x}, k_{y}\right)=\mathrm{FT}\left[E_{C}(x, y)\right]$ (where FT is the discrete Fourier transform operator).

The moving objects, that scatter the laser light, act like sources of field $S(x, y, z)$ located in volume V. According to angular spectrum propagation equations [24], the field $E_{C}(x, y)$ radiated by the sources $S$ in the plane $C$ at $z=0$ are: $E_{C}(x, y)=\sum_{z} \mathrm{FT}^{-1} \exp \left(+i k_{z} \cdot z\right) \quad \mathrm{FT}[S(x, y, z)]$, (where $\mathrm{FT}^{-1}$ is the inverse Fourier transform operator). We have thus:

$$
\tilde{E}_{C}\left(k_{x}, k_{y}\right)=\sum_{z} \exp \left(+i k_{z} \cdot z\right) \mathrm{FT}[S(x, y, z)]
$$

where $k_{z}$ is the $z$ component of the wavevector $\mathbf{k}$ i.e. $k_{z}=\sqrt{k^{2}-k_{x}^{2}-k_{y}^{2}}$ with $k=|\mathbf{k}|=2 \pi n_{m} / \lambda$, where $\lambda$ is the wavelength in vacuum and $n_{m}$ the refractive index of the medium. Equation (1) can be written as a linear system of equations:

$$
\mathbf{u}=A \mathbf{v}
$$

where $\mathbf{v} \equiv S(x, y, z)$ and $\mathbf{u} \equiv \tilde{E}_{C}\left(k_{x}, k_{y}\right)$ are vectors of dimensions 128450560 (i.e. $896 \times 896 \times 160$ and 802816 (i.e. $896 \times 896$ ), and where $A$ is a matrix of dimension $128450560 \times 802816$ (see Fig. 2 (a) ).

This system is strongly underdetermined since the dimensions of vectors $\mathbf{u}$ and $\mathbf{v}$ correspond to the number of pixels of the calculation grid in plane $C$, (i.e. $896 \times 896$ for $\mathbf{u}$ ) and the number of voxels of the volume $V$, respectively. The ratio between these two dimensions that we call the compression ratio (CR) is large, since it corresponds to the number of planes $z$ considered for the $3 \mathrm{D}$ reconstruction (i.e. 160). In the case where the moving diffusers represent a very small part of the $3 \mathrm{D}$ volume, vector $\mathbf{v}$ is highly sparse in the basis of the positions $x, y, z$. It is then possible to solve (2) using CS.

We should note that the matrix $A$ is too large to be calculated. To compute $\mathbf{u}=A \mathbf{v},(1)$ must be used. Similarly, to compute $\mathbf{v}=A^{\dagger} \mathbf{u}$, we must use (3):

$$
S(x, y, z)=\mathrm{FT}^{-1}\left[\exp \left(-i k_{z} . z\right) \tilde{E}_{C}\left(k_{x}, k_{y}\right)\right]
$$

The CS method we used here is based on the orthogonal matching pursuit [16]. The algorithm can be schematized by the equation:

$$
\mathbf{u}^{(n+1)}=\mathbf{u}^{(n)}-a_{n} A \mathrm{~T}^{(n)} A^{\dagger} \mathbf{u}^{(n)}
$$

where $n$ is the iteration index. It starts with $\mathbf{u}^{(1)}=\mathbf{u} \equiv$ $\tilde{E}_{C}$.

The algorithm calculates iteratively the $3 \mathrm{D}$ sources $\mathbf{v}^{(n)}=A^{\dagger} \mathbf{u}^{(n)}$, and the $2 \mathrm{D}$ field $A \mathbf{v}^{(n)}$ radiated by the sources, by selecting within the sources $\mathbf{v}^{(n)}$ the brightest ones with the operator $\mathrm{T}^{(n)}$. The $2 \mathrm{D}$ field radiated by the selected sources (i.e. $A \mathrm{~T}^{(n)} A^{\dagger} \mathbf{u}^{(n)}$ ) is substracted from the $2 \mathrm{D}$ field $\mathbf{u}^{(n)}$ in order to get the $2 \mathrm{D}$ field for the next iteration, i.e. $\mathbf{u}^{(n+1)}$. By this way the $2 \mathrm{D}$ field is cleaned.

(a)

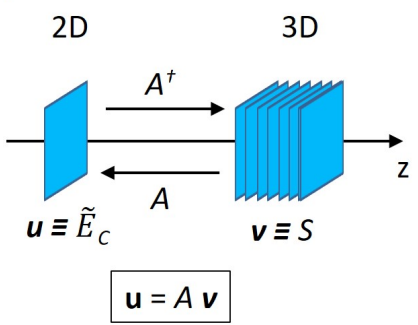

(b)

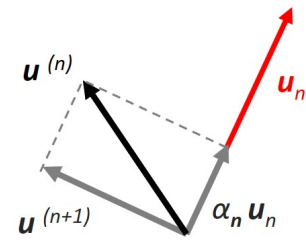

Figure 2: (a) Schematic representation of the transformations. Matrix $A$ describes the emission by the $3 \mathrm{D}$ sources $\mathbf{v} \equiv S$ resulting in a $2 \mathrm{D}$ field in Fourier space $\mathbf{u} \equiv \tilde{E}_{C}$. The matrix $A^{\dagger}$ represents the $3 \mathrm{D}$ field reconstruction from the $2 \mathrm{D}$ field in the camera conjugate plane. (b) Normalization procedure diagram : $\mathbf{u}^{(n)}$ is the hologram at the iteration $n, \mathbf{u}_{n}$ the field radiated by the sources selected at iteration $\mathrm{n}, a_{n}$ the normalization coefficient and $\mathbf{u}^{(n+1)}$ the hologram at the iteration $n+1$, orthogonal to $\mathbf{u}_{n}$.

In order to optimize and stabilize this cleaning procedure, the weight of the selected sources must by adjusted. The selected sources $\mathrm{T}^{(n)} \mathbf{v}^{(n)}$ are thus multiplied by a normalisation factor $a_{n}$, which is adjusted in order to minimize the $2 \mathrm{D}$ field energy $\left|\mathbf{u}^{(n+1)}\right|^{2}$ remaining after cleaning. To achieve this condition, the orthogonal matching method adjusts $a_{n}$ so that the vector $\mathbf{u}^{(n+1)}$ becomes orthogonal to the vector $\mathbf{u}_{n}=A \mathrm{~T}^{(n)} A^{\dagger} \mathbf{y}^{(n)}$ radiated by the selected sources (see Fig. 2 (b)). We have thus $\left(\mathbf{u}^{(n)}-a_{n} \mathbf{u}_{n}\right) \cdot \mathbf{u}_{n}=0$ where $\mathbf{.}$ is the scalar product. We get:

$$
a_{n}=\frac{\mathbf{u}_{n} \cdot \mathbf{u}^{(n)}}{\left|\mathbf{u}_{n}\right|^{2}}
$$

At each iteration step $n$, the total energy (summed over all pixels) remaining in the $2 \mathrm{D}$ field $\left|\mathbf{u}^{(n)}\right|^{2}$ decreases. When this energy becomes sufficiently low, for example when it represents only $20 \%$ of the initial energy, the iterative process stops. The final $3 \mathrm{D}$ distribution $\mathbf{v}$ of the sources is then:

$$
\mathbf{v}=\sum_{n} a_{n} \mathrm{~T}^{(n)} A^{\dagger} \mathbf{u}^{(n)}
$$


Let us give here more details on our calculations. The algorithm begins with $\mathbf{u}^{(1)}=\mathbf{u}$ :

1. Each iteration $n$ starts with the Fourier hologram $\mathbf{u}^{(n)} \equiv \tilde{E}_{C}^{(n)}\left(k_{x}, k_{y}\right)$.

2. The sources $\mathbf{v}^{(n)} \equiv S^{(n)}$ are calculated in the whole imaged volume by $(3)$ :

$$
S^{(n)}(x, y, z)=\mathrm{FT}^{-1}\left[\exp \left(-i k_{z} \cdot z\right) \tilde{E}_{C}^{(n)}\left(k_{x}, k_{y}\right)\right]
$$

3. The energy of the sources $\left|S^{(n)}(x, y, z)\right|^{2}$ is calculated for each voxel $(x, y, z)$, the maximum of energy $\mathcal{E}_{\text {max }}^{(n)}$ is determined, and a threshold of energy $\mathcal{E}_{t h}^{(n)}$ is chosen (for example $\mathcal{E}_{\text {th }}^{(n)}=0.9 \mathcal{E}_{\text {max }}^{(n)}$ ).

4. Operator $T^{(n)}$ selects the voxels $x^{\prime}, y^{\prime}, z^{\prime}$ whose energy is above the threshold $\mathcal{E}_{t h}^{(n)}$, and add to these voxels, the voxels located within a predefined radius (e.g. $r_{R B C}=3$ ) in the same $z=z^{\prime}$ plane. The selected sources $\mathrm{T}^{(n)} A^{\dagger} \mathbf{u}^{(n)} \equiv S_{n}(x, y, z)$ are thus:

- $S_{n}(x, y, z)=S^{(n)}(x, y, z)$ with $\left|S^{(n)}\left(x^{\prime}, y^{\prime}, z^{\prime}\right)\right|^{2}>$ $\mathcal{E}_{t h}^{(n)}, \sqrt{\left(x^{\prime}-x\right)^{2}+\left(y^{\prime}-y\right)^{2}} \leq r_{R B C}$ and $z=z^{\prime}$,

- and $S_{n}(x, y, z)=0$ otherwise.

5. The field $\mathbf{u}_{n} \equiv \tilde{E}_{C, n}\left(k_{x}, k_{y}\right)$ radiated by the sources $S_{n}$ in the plane $z=0$ is calculated according to (1) :

$$
\tilde{E}_{C, n}\left(k_{x}, k_{y}\right)=\sum_{z} \exp \left(-i k_{z} . z\right) \operatorname{FT}\left[S_{n}(x, y, z)\right]
$$

6. The normalisation coefficient $a_{n}$ is calculated by (5).

7. The cleaned 2D field $\mathbf{u}^{(n+1)} \equiv \tilde{E}_{C}^{(n+1)}\left(k_{x}, k_{y}\right)$, which will be used to start the next iteration, is calculated by:

$$
\tilde{E}_{C}^{(n+1)}\left(k_{x}, k_{y}\right)=\tilde{E}_{C}^{(n)}\left(k_{x}, k_{y}\right)-a_{n} \tilde{E}_{C, n}\left(k_{x}, k_{y}\right)(9)
$$

When the total energy of the $2 \mathrm{D}$ field becomes sufficiently low (for example if $\mathcal{E}_{\max }^{(n)}<0.2 \mathcal{E}_{\max }^{(1)}$ ), the $3 \mathrm{D}$ sources $\mathbf{v} \equiv S(x, y, z)$ are obtained:

$$
S(x, y, z)=\sum_{n} a_{n} S_{n}(x, y, z)
$$

We have validated our CS calculation by imaging the microcirculation in zebrafish (Danio rerio) larvae. Animals were handled according to standard procedures [23] following the National and European ethic guidelines for
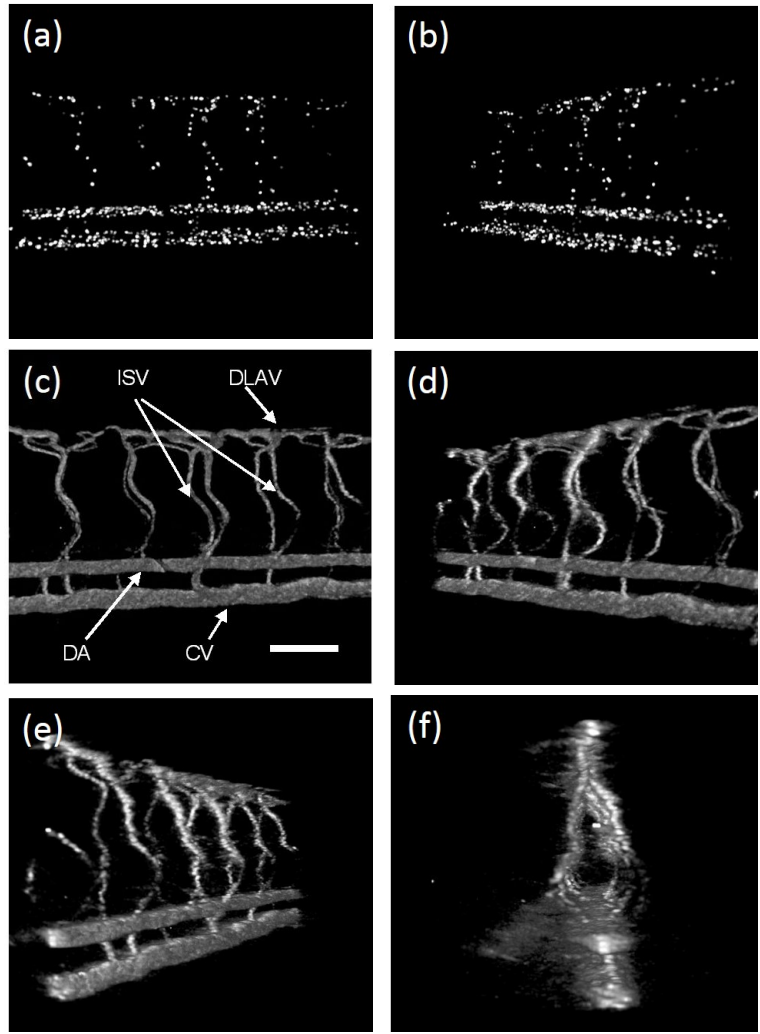

Figure 3: ( $\mathrm{a}, \mathrm{b})$ Representation of diffusers (RBCs) considering a single $3 \mathrm{D}$ reconstruction. View at $0^{\circ}$ and $+50^{\circ}$. (c,d,e,f) Representation of the vascular system considering a summation of a sequence of 100 holograms. View at $0^{\circ},+44^{\circ},-48^{\circ}$ and $-90^{\circ}$. For a $360^{\circ}$ rotation see Visualizations in supplementary. (c) DA: dorsal aorta. CV: caudal vein. ISV: intersegmental vessels. DLAV: dorsal longitudinal anastomotic vessels. Scale bar is $100 \mu \mathrm{m}$.

animal well-being and the European Convention for the Protection of Animals used for Experimental and Scientific Purposes. Unpigmented larvae were obtained from spontaneous spawning of casper young adult fish pairs and grown at $28.5^{\circ} \mathrm{C}$. They were anesthetized with tricaine at 5 days and mounted in lateral view in $1 \%$ low melting point agarose in a $35 \mathrm{~mm}$ glass ibidi $\AA$ bottom petri dish and the trunk blood flow was imaged immediately.

The experimental setup was similar to the one of previous work [10]. To increase the angular diversity of illumination, the sample was imaged with a higher NA microscope objective $(20 \mathrm{x} / \mathrm{NA}=0.5$ water immersion Zeiss microscope objective), and with three illumination beams (in place of $\mathrm{NA}=0.3$ and 2 illuminations in [10]). The holographic signal was acquired by an Andor Zyla sCMOS camera $(2560 \times 2160$ pixels, 12 Bits, 50 frames/s), 
and the frames $I_{m}$ recorded by the camera were stored on a $2560 \times 2560$ numerical grid. To select the moving RBCs, holograms are calculated by $H_{C^{\prime}}=I_{m}-I_{m-2}$. According to Verrier et al. [22], the +1 grating order signal (which corresponds to $\tilde{E}\left(k_{x}, k_{y}\right)$ and $\tilde{E}_{C}\left(k_{x}, k_{y}\right)$ ) was selected by cutting a disk of diameter 820 pixels within the Fourier space hologram $(2560 \times 2560$ pixels $)$, and by copying this disk on a $896 \times 896$ calculation grid. CS calculations were made on a $3 \mathrm{D}$ grid with $896 \times 896 \times 160$ voxels. Voxel size was $0.6 \mu \mathrm{m}$ in $x$ and $y$, and $1.8 \mu \mathrm{m}$ in $z$. The 3D view of Fig. 3, and Visualization 1 and Visualization 3 videos were obtained with ImageJ (plugin 3D Viewer). Visualization 2 and Visualization 4 videos were obtained with a home made CUDA program based on an NVidia code example (VolumeRender).

Figures 3 (a,b) show the 3D image calculated from a single hologram viewed at $0^{\circ}$ (a) and $50^{\circ}$ rotation angle (b). The individual diffusers (RBCs) are clearly seen in $3 \mathrm{D}$. A $360^{\circ}$ rotation view of the $3 \mathrm{D}$ image is shown in supplementary (Visualization $\mathbf{1}$ ). In order to visualize the $3 \mathrm{D}$ motion of the RBCs, we have calculated, from a sequence of 100 holograms, the $4 \mathrm{D}$ data corresponding to the sequence of $3 \mathrm{D}$ images. A $360^{\circ}$ rotation view of this sequence of 3D images is shown in supplementary (Visualization 2). The 3D motion of the RBCs is clearly seen. However, the RBCs movement is slightly jerky, because the camera frame rate seems too low.

The $3 \mathrm{D}$ image that is displayed in Fig. $3(\mathrm{a}, \mathrm{b})$ is highly sparse since the number of voxels whose intensity in non zero is 83268 (over the $896 \times 896 \times 160$ grid), with a relative intensity (with respect to the maximum) varying from 0.064 to 1 . The number of reconstructed voxels represents thus $15.7 \%$ of the number of measurements (i.e. of the area of the cropped disk: 528101 pixels), with most of these voxels having a very low intensity. The condition that guarantees that holographic compressive sensing 3D reconstruction is doable (number of voxels lower than $\sim 25 \%$ of the number of measurements [18]) is thus fulfilled.

The 3D calculation converge after 160 iterations (in about 2 minutes) using GPU (NVidia GTX Titan Xp) and CUDA libraries (cuFFT, cuBlas...). This is much faster than in [10], where convergence on a $512 \times 512 \times 128$ voxels grid needed about 25000 iterations. Indeed, at each iteration, both methods perform roughly the same amount of Fourier calculations $(2 \times 128$ or $2 \times 160$ FTs $)$, whereas only one voxel is extracted in [10], and about 500 voxels are extracted here. This $\sim 500 \times$ improvement of the calculation speed is made possible by the orthogonal nature of the CS method.
As was done in [10], we averaged a time sequence of 100 reconstructed 3D intensity data and got a cast of the trunk vascular system. Figures 3 (c) to (f) and Visualization 3 in supplementary show such 3D views of the vascular system. They compare favorably to confocal images of endothelial labeled larvae [13] but show only perfused blood vessels. The averaging method works here because the zebrafish larva does not move. A similar averaging method has been used by Schwerte et al. [20] to image the zebrafish vascular system in $2 \mathrm{D}$. We combined the averaged reconstructions with the ones giving instantaneous positions of the RBCs in order to visualize the blood flow inside the vessels (Visualization 4).

In this letter, we demonstrated that the $3 \mathrm{D}$ positions of small scattering objects moving in a large motionless volume can be efficiently reconstructed by a CS-based method combined with a DHM setup. The technique was applied here to the in-vivo imaging of microcirculation in a zebrafish larva, giving the instantaneous 3D positions of the red blood cells, and by averaging over a sequence of frames, the shape of the perfused vascular system. This method is however restricted to transparent animals with fairly sparse hematocrit, and to animals which do not move too much (to get the perfused vascular system by averaging).

The CS method we used here, which selects multiple sources at each iteration, is much faster $(\sim 500 \times)$ than the "cleaning" method used in [10]. Comparison with [10] shows also that the resolution is better along $z$. This improvement is mainly due to the angular diversity which is larger (larger angles, 3 illuminations), since the depth of focus is reduced accordingly.

In this work, we considered orthogonal matching pursuit which is a fairly simple CS method. More advanced CS methods like soft thresholding could be used instead [9]. It would be interesting to compare these two approaches since orthogonal matching pursuit starts from zero and adds iteratively the brightest sources, while soft thresholding removes iteratively the sources of lower energy.

We acknowledge Corinne Fournier and Laurent Daudet for fruitful discussions, and LabEx NUMEV (ANR-10LABX-20, French National Research Agency: Micro Holo 4D) and CNRS ( Défi: Instrumentation aux limites 2018 ) for funding. 


\section{References}

[1] M. Atlan, M. Gross, P. Desbiolles, E. Absil, G. Tessier, and M. Coppey-Moisan. Heterodyne holographic microscopy of gold particles. Optics letters, 33(5):500-502, 2008.

[2] D. Brady, K. Choi, D. Marks, R. Horisaki, and S. Lim. Compressive holography. Optics express, 17(15):13040-13049, 2009.

[3] E. Candes, J. Romberg, and T. Tao. Stable signal recovery from incomplete and inaccurate measurements. Communications on Pure and Applied Mathematics: A Journal Issued by the Courant Institute of Mathematical Sciences, 59(8):1207-1223, 2006 .

[4] K. Choi, R. Horisaki, J. Hahn, S. Lim, D. Marks, T. Schulz, and D. Brady. Compressive holography of diffuse objects. Applied optics, 49(34):H1-H10, 2010 .

[5] T. Colomb, E. Cuche, F. Charrière, J. Kühn, N. Aspert, F. Montfort, P. Marquet, and C. Depeursinge. Automatic procedure for aberration compensation in digital holographic microscopy and applications to specimen shape compensation. Applied optics, 45(5):851-863, 2006.

[6] E. Cuche, P. Marquet, and C. Depeursinge. Spatial filtering for zero-order and twin-image elimination in digital off-axis holography. Applied optics, 39(23):4070-4075, 2000.

[7] C. Cull, D. Wikner, J. Mait, M. Mattheiss, and D. Brady. Millimeter-wave compressive holography. Applied optics, 49(19):E67-E82, 2010.

[8] M. Debailleul, V. Georges, B. Simon, R. Morin, and O. Haeberlé. High-resolution three-dimensional tomographic diffractive microscopy of transparent inorganic and biological samples. Optics letters, 34(1):79-81, 2009.

[9] L. Denis, D. Lorenz, E. Thiébaut, C. Fournier, and D. Trede. Inline hologram reconstruction with sparsity constraints. Optics letters, 34(22):3475-3477, 2009 .

[10] D. Donnarumma, A. Brodoline, D. Alexandre, and M. Gross. 4d holographic microscopy of zebrafish larvae microcirculation. Optics express, 24(23):26887-26900, 2016.
[11] D. Donoho. Compressed sensing. IEEE Transactions on information theory, 52(4):1289-1306, 2006.

[12] J. Hahn, S. Lim, K. Choi, R. Horisaki, and D. Brady. Video-rate compressive holographic microscopic tomography. Optics express, 19(8):7289-7298, 2011.

[13] S. Isogai, M. Horiguchi, and B. M. Weinstein. The vascular anatomy of the developing zebrafish: an atlas of embryonic and early larval development. $D e-$ velopmental biology, 230(2):278-301, 2001.

[14] M. Marim, E. Angelini, J.-C. Olivo-Marin, and M. Atlan. Off-axis compressed holographic microscopy in low-light conditions. Optics letters, 36(1):79-81, 2011.

[15] M. Marim, M. Atlan, E. Angelini, and J.-C. OlivoMarin. Compressed sensing with off-axis frequencyshifting holography. Optics letters, 35(6):871-873, 2010.

[16] Y. Pati, R. Rezaiifar, and P. Krishnaprasad. Orthogonal matching pursuit: Recursive function approximation with applications to wavelet decomposition. In Signals, Systems and Computers., pages 40-44. IEEE, 1993.

[17] Y. Rivenson, A. Stern, and B. Javidi. Compressive fresnel holography. Journal of Display Technology, 6(10):506-509, 2010.

[18] Y. Rivenson, A. Stern, and J. Rosen. Reconstruction guarantees for compressive tomographic holography. Optics letters, 38(14):2509-2511, 2013.

[19] U. Schnars and W. Jüptner. Direct recording of holograms by a ccd target and numerical reconstruction. Applied optics, 33(2):179-181, 1994.

[20] T. Schwerte and B. Pelster. Digital motion analysis as a tool for analysing the shape and performance of the circulatory system in transparent animals. Journal of Experimental Biology, 203(11):1659-1669, 2000 .

[21] Y. Sung, W. Choi, C. Fang-Yen, K. Badizadegan, R. Dasari, and M. Feld. Optical diffraction tomography for high resolution live cell imaging. Optics express, 17(1):266-277, 2009.

[22] N. Verrier, D. Alexandre, G. Tessier, and M. Gross. Holographic microscopy reconstruction in both object and image half-spaces with an undistorted 
three-dimensional grid. Applied optics, 54(15):4672$4677,2015$.

[23] M. Westerfield. The zebrafish book : a guide for the laboratory use of zebrafish (Danio rerio). University of Oregon press, 2007.

[24] L. Yu and M. Kim. Wavelength-scanning digital interference holography for tomographic threedimensional imaging by use of the angular spectrum method. Optics letters, 30(16):2092-2094, 2005. 